\title{
Speech perception in congenitally deaf children receiving cochlear implants in the first year of life
}

\author{
BA Tajudeen, MD, SB Waltzman, PhD, D Jethanamest, MD, and MA Svirsky, PhD \\ Department of Otolaryngology, New York University School of Medicine
}

\section{Abstract}

Objective-1) To investigate whether children implanted in the first year of life show higher levels of speech perception than later-implanted children, when compared at the same ages, and 2) to investigate the time course of sensitive periods for developing speech perception skills. More specifically, to determine whether faster gains in speech perception are made by children implanted before 1-yr-old relative to those implanted at 2 or 3 years.

Methods-A retrospective analysis was completed on 117 patients with congenital profound bilateral sensorineural hearing loss, with no additional identified disabilities, who were implanted in the first, second, or third year of life. The outcome measure consisted of developmental curves showing LNT word identification scores as a function of age.

Results-Children implanted within the first year of life have a mean advantage of $8.2 \%$ LNT-easy word scores over those implanted in the second year $(\mathrm{p}<0.001)$ and a $16.8 \%$ advantage in LNT-easy word scores over those implanted in the third year of life $(\mathrm{p}<0.001)$. These advantages remained statistically significant after accounting for gender, residual hearing and bilateral cochlear implant use. When speech perception scores were expressed as a function of "hearing age" rather than chronological age, however, there were no significant differences among the three groups

Conclusions-There is a clear advantage for earlier implanted children over later implanted children when compared at the same age, but not when compared at the same time after implantation with regards to speech perception. Thus, the sensitive period for developing word identification seems to extend at least until age 3 .

Correspondence: Mario A. Svirsky, Dept. of Otolaryngology, NYU School of Medicine, 550 First Ave., NBV-5E5, New York, NY 10016 Fax: 212263 7604; Voice: 212263 7217; Mario.Svirsky@nyumc.org.

FINANCIAL DISCLOSURE

Intellectual Property

I am the author of a patent submitted by NYU for a new type of fitting system for cochlear implants. I have communicated to my department chair at NYU that I will donate to charity any income that I might receive in connection with this patent. This fitting system is not directly related to the subject matter of the submitted paper and is mentioned here only to be as comprehensive as possible.

Consulting

Cochlear Corp. has hired me as a consultant to advise them about the clinical trials for their new electroacoustic cochlear implant. So far, no consulting activities have taken place and therefore I have received no payments from the company. I have pledged to donate to charity any income that I receive from this consulting agreement (after taxes). The agreement has been approved by NYUSOM's committee on conflicts of interest.

This is a PDF file of an unedited manuscript that has been accepted for publication. As a service to our customers we are providing this early version of the manuscript. The manuscript will undergo copyediting, typesetting, and review of the resulting proof before it is published in its final citable form. Please note that during the production process errors may be discovered which could affect the content, and all legal disclaimers that apply to the journal pertain. 


\section{Introduction}

Mounting evidence suggests that better speech perception is achieved by congenitally deaf children who receive cochlear implants (CIs) earlier rather than later. For example, FryaufBertschy et al. (1) examined speech perception scores in children who received CIs between the ages of 2.5 and 15 years old, finding a significant advantage for those implanted earlier. In particular, the 9 children who were implanted between 2.5 and 4 years of age achieved much higher open-set speech perception scores than the 8 children who were implanted at 8 years old or later, even when comparing outcomes at the same time after implantation. This result is consistent with the existence of a sensitive period for the development of speech perception. In general, other studies $(2,3,4)$ found similar trends but there have been some exceptions $(5$, 6). Geers (5) examined 181 children who were 8 to 9 years old and who had been implanted at 2, 3 or 4 years of age. The study did not find an effect of age at implantation on speech perception, or any of the other outcome measures that were studied (speech production, language, and reading). Geers indicated that "further research examining the benefits of cochlear implantation before age 2 years will help families and clinicians better understand the time-sensitive nature of the decision to conduct cochlear implant surgery". Holt and Svirsky (6) used Developmental Trajectory Analysis, a novel data analysis method to examine the effect of age at intervention (7). This method was used to study speech perception scores in children implanted in the first, second, third, or fourth year of life and did find a general advantage in speech perception scores for earlier implanted children (when compared at the same age). The only exception was the relatively small group of six children implanted in the first year, whose performance was not significantly different from those implanted in the second year. As in the case of speech perception studies, examination of other outcome measures also has generally shown that earlier implanted children tend to have more intelligible and/or accurate speech production $(8,9,10)$ and higher levels of language development $(7,11$, $12,13,14,15)$.

As clearly discussed by Tomblin et al. (16), there are at least two ways in which earlier implantation can result in better outcomes. First, early implantation may simply allow children to get a head start even if the growth rate of the outcome measure is not affected. Second, the growth rate itself may be higher for children who are implanted earlier. The latter situation may happen when there is a time during development when a child is more responsive to experience and a given skill is learned most efficiently. This special time in development is called "sensitive period", and there is some evidence that sensitive periods exist for the development of intelligible speech production (10) and language skills in children with cochlear implants $(11,13,14,15)$. Some of these studies have found differences in growth rates between children implanted in the first and the second, or the second and third years of life. For example, Dettman et al. (15) followed receptive and expressive language growth for 19 infants and 87 toddlers who received a multichannel implant. They showed that language growth for children receiving implants before the age of 12 mo were significantly greater than rates achieved by children receiving implants between 12 and $24 \mathrm{mo}$, and matched growth rates achieved by normally hearing peers. Evidence for a relatively weak sensitive period for the development of intelligible speech was found by Svirsky et al. (10), who observed higher average growth rates in this outcome measure in children implanted before their second birthday than in children implanted later. However, and despite the primary importance of speech perception as an outcome of pediatric cochlear implantation, the time course of the sensitive period for the development of word identification skills has not been established.

Widespread use of universal newborn hearing screening programs, advances in technology and increased general awareness of cochlear implantation have resulted in increasing numbers of children younger than 12 months of age being considered for cochlear implantation, even though this is considered an off-label use of CIs by the Food and Drug Administration at this 
time. In the present study a retrospective analysis was completed on 117 patients with congenital profound sensorineural hearing loss bilaterally with no additional identified disabilities who were implanted within the first three years of life. Subjects were stratified into three groups based on age at implantation. Word identification scores were obtained from sequential testing dates and used to create developmental curves showing speech perception as a function of time. The aims of the present study were to investigate whether children implanted in the first year of life show higher levels of speech perception than children who are implanted later, when compared at the same ages, and to investigate whether faster gains in speech perception are made by children implanted before 1-yr-old relative to those implanted at 2 or 3 years. To our knowledge, the present study is the first one to compare speech perception development in children implanted within the first year of life to children implanted later, using a large number of subjects ( $\mathrm{N}=37$ implanted in the first year, $\mathrm{N}=117$ total). This type of analysis is necessary due to the growing importance of this population (children implanted in the first year) and the crucial nature of speech perception as a principal outcome of cochlear implantation.

\section{Participants}

The children in this study included all pediatric CI recipients followed longitudinally between June 1993 and September 2008 at the authors' institution, subject to the following inclusion and exclusion criteria: subjects had bilateral severe-to-profound sensorineural hearing loss $($ PTA $>=80 \mathrm{~dB})$ and derived minimal or no benefit from conventional amplification, subjects had no additional identified disability, had been implanted with a current device before the age of 3yrs, and made use of spectral peak (SPEAK), Continuous Interleaved Sampling (CIS), or Advanced Combination Encoding (ACE) stimulation strategies since initial fitting.

Participants spoke English and were evaluated using at least one of the speech perception tests described below. This resulted in a total of 117 children. The children were stratified into three groups based on age at implantation. Group 1 was implanted between 6 and 12 months of age, group 2 was implanted between 13 and 24 months of age, and group 3 between 25 and 36 months of age. Table 1 displays demographic information for each group, including number of participants, mean age at implantation, number of subjects with bilateral implants, mean unaided best-ear pure tone average, and proportion of females.

\section{Methods}

Open-set speech perception tests were performed in a sound-proof booth using recorded material without repetition. The cochlear implant setting was not readjusted or reprogrammed before testing and was fixed at the program used routinely by the subject. The speakers used were GSI high-performance sound field loudspeakers mounted $1 \mathrm{~m}$ in front of the subject. Children were tested before cochlear implantation, three months after implantation and at regular $6 \mathrm{mo}$ to $1 \mathrm{yr}$ intervals thereafter. Not all children were tested at every interval because of time constraints, lack of ability to maintain attention for all tests, and missed appointments. The test materials were administered and scored by licensed audiologists.

Given the wide range of ages and abilities of the pediatric subjects, age- and skill-appropriate speech recognition tests were used. The Glendonald Auditory Screening Procedure (GASP) consists of 12 words and 10 questions. The child is required to repeat the words and the test is scored as a percent correct (17). The Multisyllable Lexical Neighborhood test (MLNT) consist of lists of 15-item "easy" (level 1) and 15-item "hard" (level 3) two- to three-syllable words and is scored as a percent correct (18). The Lexical Neighborhood test (LNT) consists of two "easy" 25-item monosyllabic word lists (level 1) and two more difficult 25-item monosyllabic word lists (level 3) and is scored as a percent correct (18). The Phonetically Balanced Kindergarten (PBK) test consists of 50 monosyllabic words. The child is required to repeat the 
stimulus presented and the score is the percent of words and phonemes correctly repeated (19). The Common Phrases test is a sentence test designed for young children. There are six lists, which consist of 10 questions or statements per list. The child is asked to repeat the stimulus, and the score is based on percent correct (20).

\section{Prediction of LNT score}

The main goal of the present study was to evaluate the development of speech perception skills over time as a function of age at implantation. The LNT-easy was selected as the main outcome measure to create developmental curves showing speech perception as a function of time because most of the data for the study were obtained using this widely accepted test. However, different speech recognition tests (other than the LNT-easy) were used at some of the testing sessions. In these cases, when LNT-easy scores were not available for a specific testing date, scores were predicted based on the other speech perception tests given on that testing date. The predictive functions were based on linear regression analyses done using scatterplots of LNTeasy scores as a function of one of the other measures: GASP words, MLNT hard words, Common Phrases sentences in quiet, LNT hard words and PBK words score. Each data point within a scatter plot represents scores obtained using two different tests at the same testing session. Data points in which any test score was either 0 or 100 were deemed uninformative and not included in the linear regression. The number of data points used in each analysis is shown in Table 2.

\section{Developmental Trajectory Analysis}

Developmental curves were created by plotting LNT-easy word scores versus chronological age at testing. When more than one test was available to predict LNT-easy score, the test that was most highly correlated with LNT-easy was utilized. Patients with bilateral implants receive testing in three conditions (the first implanted ear alone, the second implanted ear alone, and the bilateral condition). For the present study, LNT-easy scores of the first implanted ear were used in the analyses so as to minimize the influence of the second implant on the results. Developmental Trajectory Analysis (6) was used to determine whether the average developmental curve for a given age-at-implant group shows better scores than the curve for another age-at-implant group. This analysis method was also used to calculate the average difference among groups throughout their post-implant period, and to factor out the effect of some potential confounding variables, including gender, the use of bilateral implants, and the amount of residual hearing. An important characteristic of Developmental Trajectory Analysis (DTA) is that the outcome measures are whole developmental curves obtained over time (typically several years) rather than data obtained at a single point in time. DTA answers the following questions: when we compare $\mathrm{N}$ groups of subjects as they make progress over time, which group outperforms the other over the entire age range, as both groups grow older? What is the size of the difference between average developmental curves? Are these differences statistically significant? The comparisons are made at the same ages, as all groups grow older.

In order to investigate the time course of the sensitive period for speech perception acquisition at a group level, average developmental curves were generated for each one of the three ageat-implant cohorts expressing LNT-easy scores as a function of time following cochlear implantation ("hearing age"). Subtracting age at cochlear implantation from chronological age at testing generated this "hearing age" parameter utilized in the analysis. A sensitive period for the first year of life would be noted if faster gains in speech perception were made by children implanted before 1-yr-old relative to those implanted at 2 or 3 years. Conversely, if all three groups made similar gains in speech perception after implantation, this result would support the hypothesis that the sensitive period for word identification extends beyond the first three years of life. 


\section{Other statistical analyses}

One-way ANOVA was used to compare scores at a given chronological age. When the data were not normally distributed, non-parametric ANOVA on ranks was used instead.

Comparisons were made at three, four, five, and six years of age. Similar comparisons were carried out at a given "hearing age". Because all subjects were congenitally deaf, hearing age is equal to time after initial stimulation with the cochlear implant. Data were compared one, two, three, four, and five years after initial stimulation. The number of data points after five years post-implantation was too small to conduct meaningful comparisons.

\section{Results}

\section{Prediction of LNT-easy word scores}

Figure 1 shows one representative scatter plot illustrating how PBK word scores were used to predict LNT easy word scores using linear regression. The correlation between the two variables was quite strong $(\mathrm{r}=+0.86, \mathrm{p}<0.001)$, supporting the idea that LNT scores can be predicted (if necessary) based on PBK scores. Even when removing the outlier point at the bottom left of Fig. 1, the correlation remains strong and significant $(r=+0.83, p<0.001)$ Results of other linear regression analyses are depicted in Table 2. The PBK test was most highly correlated with the LNT-easy test followed by LNT-H, MLNT-H, CP-Q and the GASP test in descending order. The last column of table 2 shows how frequently the LNT scores had to be predicted based on other scores.

\section{Developmental Trajectory Analysis}

Figure 2 displays individual LNT-easy word score performance for children from each age-atimplantation group. Individually, most children in each group reached scores of $85 \%$ correct or higher by four years after implantation. The top panel of Figure 3 shows the average performance of each group. As the age increases, each curve represents the average of fewer subjects, due to different amounts of follow-up. Curves are drawn with a thinner line once they represent the average of 5 or fewer subjects, as a visual reminder that the average becomes less reliable at that point. Average data from children with normal hearing (obtained by Kluck et al. (24)) are indicated by triangles. The LNT-easy word score data for the three age-at-implant groups were entered into DTA analysis. DTA revealed that those implanted within the first year of life have a statistically significant $(\mathrm{p}<0.001)$ mean advantage of $8.2 \%$ LNT-easy word scores over those implanted in the second year and a statistically significant $(p<0.001) 16.8 \%$ advantage in LNT-easy word scores over those implanted in the third year of life. Furthermore, children implanted in the second year of life have a statistically significant $(p<0.001) 9.2 \%$ advantage in LNT-easy word score over those implanted in the third year of life.

The covariates (BPTA, bilateral use, gender) were entered into a stepwise multiple linear regression to determine if they could account for any additional variability in average developmental difference beyond age-at-implantation. Residual hearing (BPTA) and bilateral use did not significantly account for any additional variability in mean LNT-easy word between age-at-implant groups. However, the proportion of females did significantly account for variability in mean LNT-easy word score when comparing those implanted within the first year of life to those implanted in the second year of life $(\mathrm{p}<0.047)$ and when comparing those implanted in the first year of life to those implanted in the third year of life $(\mathrm{p}<0.043)$. After accounting for covariates, those implanted within the first year of life still have a statistically significant $(\mathrm{p}<0.001)$ mean advantage of $9 \%$ LNT-easy word scores over those implanted in the second year and a statistically significant $(\mathrm{p}<0.001) 16.4 \%$ advantage in LNT-easy word scores over those implanted in the third year of life. Likewise, children implanted in the second year of life have a statistically significant $(\mathrm{p}<0.001) 8.7 \%$ advantage in LNT-easy word score over those implanted in the third year of life after controlling for covariates. The bottom panel 
of Figure 3 shows LNT-easy scores as a function of time following cochlear implantation ("hearing age"). Visual inspection reveals that the average performance curves of the three age-at-implant groups superimposes almost exactly during the first four years postimplantation.

\section{Comparisons at a given chronological age or hearing age}

The top panel of figure 4 shows box-whisker plots of speech perception data obtained at three, four, five, and six years of age. The asterisks on top of the bars indicate significant differences $(\mathrm{p}<0.05)$ among groups. There was a strong trend towards better performance for earlier implanted children. In particular, children implanted at 6-12 months significantly outperformed children implanted at 25-36 months not only when the comparison was done at three years of age, but also when it was done at four, five, or six years old. They also outperformed children implanted at 13-24 months when compared at three years old. Lastly, children implanted at 13-24 months did significantly better than children implanted at 25-36 months in the comparison conducted at four years of age.

The bottom panel shows comparisons conducted at the same hearing age (or, equivalently, at the same time post-implant). There was almost complete overlap in scores (and no statistically significant differences) among all three age-at-implant groups at each one of the post implant intervals: one, two, three, four, and five years post-implant.

\section{Discussion}

The first goal of this study was to explore whether higher scores in speech perception are attained the earlier a child is implanted, particularly in the case of children implanted in the first 12 months of life. Results show this is indeed the case when comparing word identification scores across a wide range of chronological ages and while controlling for potential covariates such as residual hearing, bilateral cochlear implant use and gender. These findings differ from a previous pilot study (6) that did not find a difference in speech perception scores between children implanted in the first year of life and those implanted in the second year of life. The discrepancy may be due to the small sample size of children $(\mathrm{N}=6)$ in the earlier study. An additional important point to be made is that the large advantage in speech perception scores for early implanted children in the present study should not be interpreted as precluding cochlear implantation at later ages. That is a decision that has to be made based on the expected outcomes for the relevant age-at-implantation group and, in fact, studies have shown that lateimplanted congenitally deaf children and adults can obtain substantial benefit from their devices $(21,22)$.

Our second goal was to investigate the time course of the sensitive period for speech perception development that has been found in previous studies of pediatric cochlear implantation (1). In particular, we aimed to determine whether the influence of auditory experience on the developmental trajectory of speech perception skills depends on whether such experience starts in the first, second, or third years of life, a result that would parallel similar findings for the development of language and speech production skills $(10,11,13,14,15)$. Prelingually deafened children, because of their early onset of deafness, provide a unique opportunity to study the effects of spoken language deprivation and subsequent intervention. In the present study, when comparing group averaged speech perception scores as a function of "hearing age" across the three age-at-implant groups, it was noted that the three group averaged curves superimpose almost perfectly for four years after implantation, and there were no statistically significant differences among groups during the first five years post-implant. In other words, the groups of children implanted in the first, second and third years of life made similar speech perception gains after implantation. Taken together, the present results and those from other studies (2, 6) suggest that the sensitive period for speech perception development extends at least until 
age four but definitely not until age eight. This result is in contrast with the much earlier sensitive periods found in the same population for the development of language (15) and intelligible speech production (10). One important caveat when interpreting all these studies is that the amount of exposure to spoken language and explicit rehabilitation provided to the children, either before or after implantation, was not controlled directly. As Tomblin et al. point out (16), "we need to recognize that children implanted at later ages may engage in different kinds of interaction patterns with their caregivers than children who are implanted at younger ages". Nevertheless, the study of possible differences in the time courses of sensitive periods for different communicative skills is worthy of further investigation, both for its basic scientific implications and its clinical implications.

The present speech perception data suggest there is a benefit to implanting children within the first year of life. Not only do children implanted in the first year of life attain high speech perception scores earlier, evidence also supports improved receptive and expressive language growth with minimal anesthetic, surgical, and long term complications $(10,15,22)$. However, all three groups in our study performed well over time reaching an average of about $80 \%$ correct in the LNT word identification test four years after implantation. This suggests that even though earlier implantation is beneficial, speech perception skills in groups of children implanted in the second or third year of life do catch up with those of their earlier implanted peers.

\section{Acknowledgments}

This research was partially supported by NIH grant R01-DC03937 and Dr. Waltzman's participation was partially supported by the Rienzi Foundation for Cochlear Implant Research. The authors gratefully acknowledge the assistance of the Audiology Staff at the NYU Cochlear Implant Center without whose help this work could not have been done.

As indicated above, this work was partially funded by the National Institutes of Health.

The senior author (Svirsky) has received technical and logistical support for his research from Cochlear Americas and Advanced Bionics Corporation over the last five years. He has a consulting agreement with Cochlear Americas but not consulting activities have taken place, and he has pledged to donate to charity the after-tax proceeds of any future consulting activities.

$\underline{\text { Research Support }}$

Over the last five years I have received software, equipment donations, and technical support from two cochlear implant companies (Advanced Bionics Corp. and Cochlear Corp.) and one workstation manufacturer (Sun Microsystems).

$\underline{\text { Grants }}$

My cochlear implant research is supported by a grant from NIH, in which I am the Principal Investigator.

My academic department had a research contract with Advanced Bionics Corporation (2005-2008). I was neither the Principal Investigator nor a named investigator in this contract

Mario A. Svirsky

\section{References}

1. Fryauf-Bertschy H, Tyler RS, Kelsay DMR, et al. Cochlear implant use by prelingually deafened children: the influences of age at implant and length of device use. J Speech Lang Hear Res 1997;40 (1):183-99. [PubMed: 9113869]

2. Dawson PW, Blamey PJ, Rowland LC, et al. Cochlear implants in children, adolescents, and prelinguistically deafened adults-speech-perception. J Speech Hear Res 1992;35:401-417. [PubMed: 1573879]

3. Nikolopoulos TP, O'Donoghue GM, Archbold S. Age at implantation: its importance in pediatric cochlear implantation. Laryngoscope 1999;109(4):595-9. [PubMed: 10201747] 
4. Kirk KI, Miyamoto RT, Lento CL, et al. Effects of age at implantation in young children. Ann Otol Rhinol Laryngol Suppl 2002;189:69-73. [PubMed: 12018353]

5. Geers AE. Speech, language, and reading skills after early cochlear implantation. Arch Otolaryngol Head Neck Surg 2004;130:634-638. [PubMed: 15148189]

6. Holt RF, Svirsky MA. An exploratory look at pediatric cochlear implantation: is earliest always best? Ear Hear 2008;29(4):492-511. [PubMed: 18382374]

7. Svirsky MA, Teoh SW, Neuburger H. Development of language and speech perception in congenitally, profoundly deaf children as a function of age at cochlear implantation. Audiol Neuro-Otol, 2004;9(4): 224-233.

8. Osberger MJ, Maso M, Sam LK. Speech intelligibility of children with cochlear implants, tactile aids, or hearing aids. J Speech Hear Res 1993;36:186-203. [PubMed: 8450658]

9. Tye-Murray N, Spencer L, Woodworth GG. Acquisition of speech by children who have prolonged cochlear implant experience. J Speech Hear Res 1995;38:327-337. [PubMed: 7596098]

10. Svirsky MA, Chin SB, Jester A. The effects of age at implantation on speech intelligibility in pediatric cochlear implant users: Clinical outcomes and sensitive periods. Audiol Med 2007;5(4):293-306.

11. Connor CM, Hieber S, Arts HA, Zwolan TA. Speech, vocabulary, and the education of children using cochlear implants: Oral or total communication? J Speech Lang Hear Res 2000;43:1185-1204. [PubMed: 11063240]

12. Svirsky MA, Teoh SW, Neuburger H. Development of language and speech perception in congenitally, profoundly deaf children as a function of age at cochlear implantation. Audiol NeuroOtol 2004;9(4):224-233.

13. Tomblin JB, Barker BA, Spencer LJ, Zhang X, Gantz BJ. The Effect of Age at Cochlear Implant Initial Stimulation on Expressive Language Growth in Infants and Toddlers. J Speech Lang Hear Res 2005;48:853-867. [PubMed: 16378478]

14. Nicholas JG, Geers AE. Will they catch up? The role of age at cochlear implantation in the spoken language development of children with severe to profound hearing loss. J Speech Lang Hear Res 2007;50(4):1048-62. [PubMed: 17675604]

15. Dettman SJ, Pinder D, Briggs RJ, Dowell RC, Leigh JR. Communication development in children who receive the cochlear implant younger than 12 months: risks versus benefits. Ear Hear 2007;28 (2 Suppl):11S-18S. [PubMed: 17496638]

16. Tomblin J. Bruce, Barker, Brittan A. and Hubbs, Sarah. Developmental constraints on language development in children with cochlear implants. Int J Audiol 2007;46(9):512-523. [PubMed: 17828667]

17. Erber N. Glendonald auditory screening procedure. Auditory training 1982;47(71)

18. Kirk KI, Pisoni DB, Osberger MJ. Lexical effects on spoken word recognition by pediatric cochlear implant users. Ear Hear 1995;16(5):470-81. [PubMed: 8654902]

19. Haskins, H. A phonetically balanced test of speech discrimination for children. 1949. unpublished master's thesis

20. Robbins A, Renshaw JJ, Osberger MJ. Common phrases (test). Indiana University School of Medicine. 1988

21. Waltzman SB, Cohen NL. Implantation of patients with prelingual long-term deafness. Ann Otol Rhinol Laryngol Suppl 1999;177:84-7. [PubMed: 10214808]

22. Waltzman SB, Roland JT Jr. Cohen NL. Delayed implantation in congenitally deaf children and adults. Otol Neurotol 2002;23(3):333-40. [PubMed: 11981391]

23. Roland JT Jr, Cosetti M, Wang KH, et al. Cochlear implantation in the very young child: Long-term safety and efficacy. Laryngoscope 2009;119(11):2205-2210. [PubMed: 19507225]

24. Kluck M, Pisoni DB, Kirk KI. Performance of Normal-Hearing Children on Open-Set Speech Perception Tests. Research on Spoken Language Processing 1997:349-366. Progress Report No. 21. 


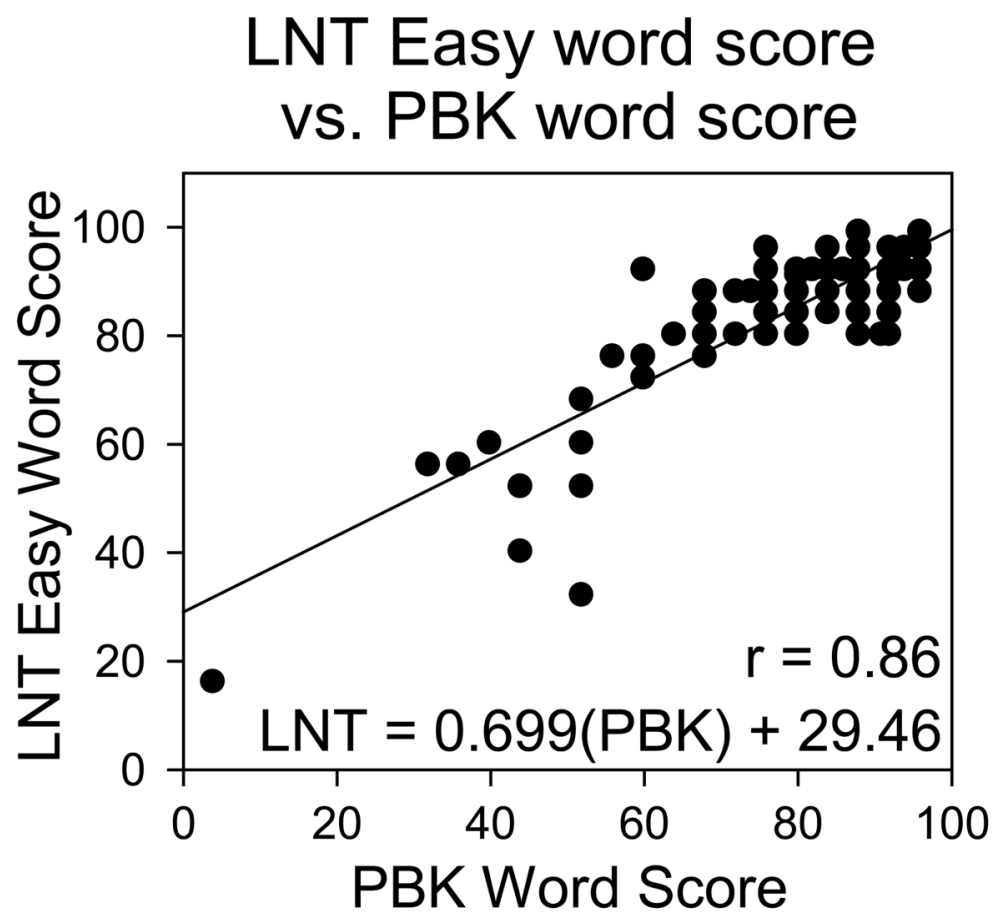

Figure 1.

Scatterplot displaying LNT Easy word score as a function of PBK word score. 


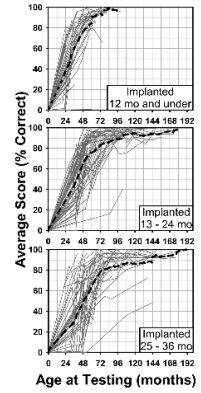

Figure 2.

Individual (thin lines) and group-averaged (dashed line) LNT-easy word scores for each ageat-implant group: 12 months and under (top panel), 13-24 months (middle panel) and 25-36 months (bottom panel). 

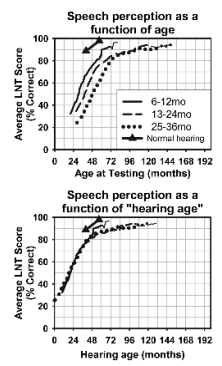

Figure 3.

Top panel shows average LNT Easy word score for each age-at-implant group as a function of chronological age at testing. Bottom panel shows average LNT Easy word score as a function of "hearing age" (i.e., time following cochlear implantation) for each age-at-implant group. Average performance curve thickness is decreased once the number of patients used to compute the average falls below 5 . Triangles indicate average scores obtained from children with normal hearing. 

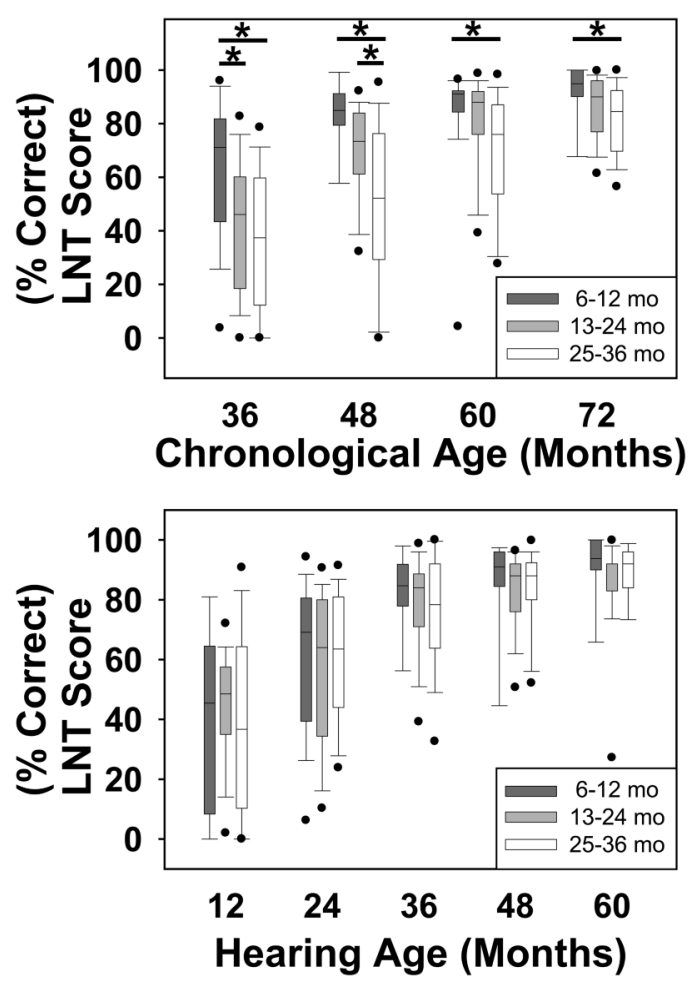

Figure 4.

Box and whisker plots showing LNT scores for each age-at-implant group at a given chronological age (top panel) or hearing age (bottom panel), Asterisks indicate significant differences between groups $(\mathrm{p}<0.05)$. 


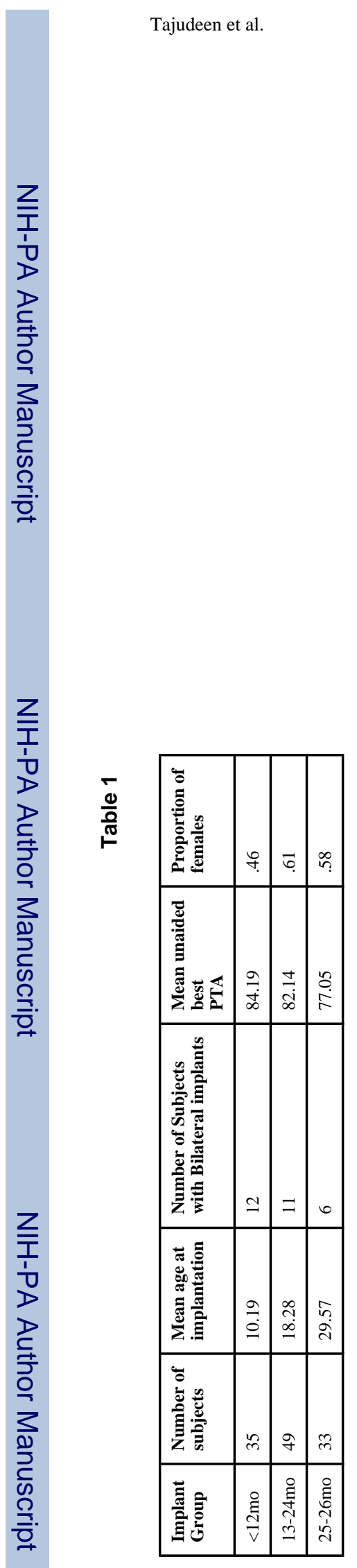

Page 13

\begin{tabular}{|c|c|c|c|}
\hline 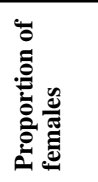 & q. & $\overline{6}$ & $\stackrel{\infty}{n}$ \\
\hline 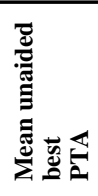 & $\stackrel{\overrightarrow{+}}{\dot{+}}$ & \begin{tabular}{|l}
$\underset{i}{i}$ \\
$i$ \\
$\infty$
\end{tabular} & 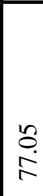 \\
\hline 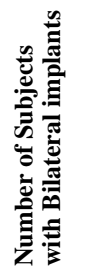 & $\simeq$ & $=$ & 0 \\
\hline 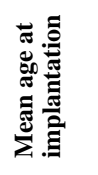 & $\stackrel{\circ}{\stackrel{0}{\Theta}}$ & \begin{tabular}{|l}
$\stackrel{\infty}{\sim}$ \\
$\stackrel{\infty}{\longrightarrow}$
\end{tabular} & $\begin{array}{l}\hat{n} \\
\text { ते }\end{array}$ \\
\hline 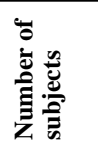 & 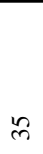 & $g$ & mे \\
\hline 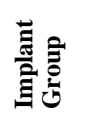 & $\begin{array}{l}\stackrel{g}{\mathrm{~d}} \\
\stackrel{\mathrm{v}}{\mathrm{v}}\end{array}$ & 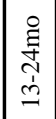 & 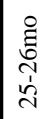 \\
\hline
\end{tabular}

Otol Neurotol. Author manuscript; available in PMC 2011 October 1. 


\begin{tabular}{|c|c|c|c|c|c|}
\hline 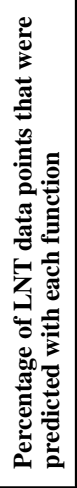 & \begin{tabular}{|l} 
\\
$\therefore$
\end{tabular} & $\stackrel{\circ}{\stackrel{2}{n}}$ & $\stackrel{\circ}{\stackrel{0}{0}}$ & in & $\stackrel{\circ 0}{I}$ \\
\hline & 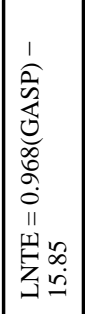 & 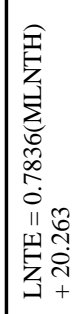 & 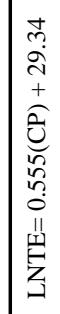 & 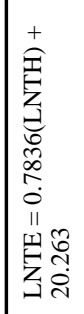 & 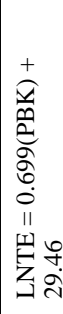 \\
\hline ¿ & 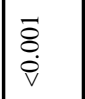 & $\begin{array}{l}\overrightarrow{8} \\
\dot{\nabla} \\
\dot{\nabla}\end{array}$ & $\begin{array}{l}\vec{\delta} \\
\dot{\sigma} \\
\dot{\nabla}\end{array}$ & $\begin{array}{l}\vec{\delta} \\
\dot{\sigma} \\
\dot{\nabla}\end{array}$ & $\begin{array}{l}\overrightarrow{8} \\
\stackrel{+}{v}\end{array}$ \\
\hline 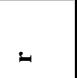 & $\tilde{n}$ & î & in & $\mid \begin{array}{l}+ \\
\infty \\
0 \\
0\end{array}$ & $\begin{array}{l}0 \\
\infty \\
0\end{array}$ \\
\hline z & f & 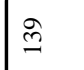 & $\approx$ & $\vec{m}$ & సิ \\
\hline$\underset{f}{\vec{s}}$ & $\begin{array}{l}\hat{\vec{z}} \\
\text { s. }\end{array}$ & $\left.\right|_{\text {岁 }} ^{\text {岁 }}$ & $\begin{array}{l}o \\
o \\
\dot{u}\end{array}$ & 蒋 & \\
\hline
\end{tabular}

Otol Neurotol. Author manuscript; available in PMC 2011 October 1. 